Original article

\title{
Prostaglandin D2 receptor D-type prostanoid receptor 2 mediates eosinophil trafficking into the esophagus
}

\author{
S. Zhang, X. Wu, S. Yu \\ Division of Gastroenterology, Department of Medicine, University of Michigan Medical School, Ann Arbor, \\ Michigan, USA
}

\begin{abstract}
SUMMARY. Eosinophilic esophagitis is characterized by eosinophil-predominant inflammation in the esophagus. How eosinophils migrate and infiltrate into the esophagus, however, is less clear. Our previous study demonstrated that mast cell activation led to eosinophil infiltration in the esophagus. Prostaglandin D2 (PGD2) is an important mediator released from activated mast cells. The present study aims to determine whether PGD2 induces eosinophil infiltration into the esophagus via a D-type prostanoid receptor 2 (DP2) receptor-dependent mechanism. Using an in vivo guinea pig model, PGD2, D-type prostanoid receptor 1 (DP1) agonist, or DP2 agonist were injected into the esophagus. Esophageal tissues were removed 2 hours after injections and proceeded to either hematoxylin-eosin (HE) staining or immunofluorescent staining of esinophil major basic protein (MBP) to compare each treatmentinduced eosinophil infiltration in the esophagus. In a separate study, ovalbumin (OVA)-sensitized guinea pigs were pretreated with either DP2 or DP1 antagonists, followed by inhalation of OVA to induce mast cell activation. Esophageal tissues were then processed for immunofluorescent staining of MBP. PGD2 injection in the esophagus led to an increase of eosinophil infiltration in esophageal epithelium at the injection site as revealed by HE staining. Increased infiltration of eosinophils was further confirmed by the increased presence of MBP-labeled immunopositive (MBP-LI) cells in esophageal epithelium. Injection with DP2 agonist 15(R)-PGD2, but not DP1 agonist BW 245C, mimicked the PGD2-induced response. In OVA-sensitized animals, antigen inhalation increased MBP-LI cells in esophageal epithelium. Pretreatment with DP2 antagonist BAY-u3405, but not DP1 antagonist BW 868C, inhibited the antigen inhalation-induced increase of MBP-LI cells in esophageal epithelium. These data support the hypothesis that PGD2 induces eosinophil trafficking into the esophageal epithelium via a DP2mediated pathway, suggesting a role of DP2 antagonist in the prevention of essinophilic esophagitis.
\end{abstract}

KEY WORDS: eosinophilic esophagitis (EoE), mast cell, prostaglandin D2.

\section{INTRODUCTION}

Eosinophilic esophagitis (EoE) has emerged in the last two decades as a significant esophageal disorder affecting all age groups. ${ }^{1}$ In esophageal biopsy, 15 eosinophils per high-power field under microscope are considered a minimum threshold for the diagnosis of EoE. ${ }^{1,2}$ Eosinophils are derived from CD $34+$ hematopoietic progenitor cells in the bone marrow and are found mainly in circulation rather

Address correspondence to: Professor Shaoyong Yu, MD, MPH, Division of Gastroenterology, Department of Medicine,

University of Michigan Medical School, Ann Arbor, MI 48105,

USA. Email: syyu@jhmi.edu

Grant Support: National Institute of Health (NIH)

grant-DK087991

Disclosure: The authors have nothing to disclosure. than in peripheral tissues. Normal human esophageal tissue has only minimal eosinophils, ${ }^{3}$ but their number increased in the inflamed esophagus. ${ }^{4}$ Even though increased infiltration and degranulation of eosinophils are the predominant features of EoE, other inflammatory cells such as mast cells and T-helper cells may also play important roles in the development of EoE. Currently, the mechanisms of eosinophil migration from the circulation with infiltration into the esophagus are still less clear. Previous studies demonstrated that certain Th2 cytokines and chemokines such as interleukin (IL)-5, IL-13, and eotaxin play important roles in this infiltration process. ${ }^{1}$ A recent clinical trial revealed that anti-IL-5 antibody therapy significantly reduces, but does not normalize, esophageal eosinophil infiltration in patients with EoE, and clinical symptom 
improvement is not as promising ${ }^{5}$ as diet elimination. ${ }^{6}$ This suggests that factors other than the Th2 cytokine IL-5 may also contribute to this infiltration process.

Recently, several studies have demonstrated that there are increased numbers of mast cells in the esophagus in patients with EoE. ${ }^{7-10}$ An important human subject study revealed that esophageal mast cell degranulation and mastocytosis significantly increased in patients with EoE, and such increase was correlated with the expression of transcripts for mast cell proteases carboxypeptidase A3 and tryptase. ${ }^{10}$ This suggests that mast cells may play an important role in the development of EoE. Activated mast cells not only release preformed mediators such as histamine and tryptase, but also produce de novo synthesized lipid mediators including prostaglandin D2 (PGD2) and leukotrienes D4, etc. Both histamine and PGD2 are identified to play important roles as chemoattractants to induce eosinophil infiltration into the airway of patients with asthma. Our previous study demonstrated that mast cell preformed mediator histamine plays an important role in mast cell activation-induced eosinophil infiltration in the esophagus. ${ }^{11}$ In the present study, we aimed to determine the role of PGD2 in mast cell activationinduced infiltration of eosinophils in the esophagus.

The biological effects of PGD2 are usually mediated by its two G-protein-coupled receptors: D-type prostanoid receptor 1 (DP1) and D-type prostanoid receptor 2 (DP2) (also known as CRTH2: chemoattractant homologous receptor expressed on Th2 cells). We hypothesized that PGD2 could induce eosinophil infiltration in the esophagus via a DP2dependent mechanism. Our present data demonstrated that PGD2 injection into the esophagus leads to an increased infiltration of eosinophils in the esophagus. DP2 agonist was able to mimic this effect. Pretreatment with DP2 antagonist prevented mast cell activation-induced infiltration of eosinophils in the esophagus. This result provided the first evidence that mast cell mediator PGD2 plays an important role in eosinophil trafficking into the esophagus.

\section{MATERIALS AND METHODS}

Male Hartley guinea pigs (Hilltop Laboratory Animals, Inc., Scottsdale, PA, USA) weighing 100$300 \mathrm{~g}$ were used. All experiments were approved by the University of Michigan Committee on Use and Care of Animals.

\section{PGD2 and agonists injections in the esophagus}

Naive guinea pigs were anesthetized by intraperitoneal (IP) injection of urethane $(1.1 \mathrm{mg} / \mathrm{kg})$. The cervical esophagus was surgically exposed; PGD2 $(n=4)$, DP2 agonist $15(\mathrm{R})-\mathrm{PGD} 2(n=3)$, or DP1 agonist BW 245C $(n=3)$ were injected (each with $20 \mu \mathrm{L}$ at $100 \mathrm{nM}$ ) into the wall of the esophagus (70 $\mathrm{mm}$ from incisors), respectively. Control animals received phosphate-buffered saline (PBS) injections. Two hours after injections, animals were killed by $\mathrm{CO}_{2}$ inhalation and exsanguination. The esophagi were removed, processed, and sectioned for HE staining or immunofluorescent staining of major basic protein (MBP).

\section{Active immune sensitization and allergen challenge}

In a separate study, guinea pigs were actively sensitized and challenged by allergen as previously described. ${ }^{11}$ Briefly, guinea pigs were given three IP injections of ovalbumin (OVA, $10 \mathrm{mg} / \mathrm{kg}$ ) every 48 hours. Three weeks after the last injection, guinea pigs were exposed to aerosolized antigen $(0.1 \%$ OVA $)$ in a plastic chamber for 2-10 minutes depending on the development of dyspnea. The OVA was dissolved in $0.9 \%$ saline and delivered using a nebulizer driven by compressed air. As control, sensitized animals were exposed for 10 minutes to aerosolized $0.9 \%$ saline by the same technique. The guinea pigs were closely monitored for signs of any allergic responses such as gasping or increased respiratory rate. Once guinea pigs developed such responses, they were removed and allowed to breathe ambient air. Animals were euthanized by $\mathrm{CO}_{2}$ inhalation and exsanguination 2 hours after OVA challenge. Two additional groups of sensitized animals received intravenous injection of either BAY-u3405 (DP2 receptor antagonist, at a dose of $5 \mathrm{mg} / \mathrm{kg}$, Cayman Chemicals, Ann Arbor, MI, USA) or BW A868C (DP1 antagonist, at a dose of $0.1 \mathrm{mg} / \mathrm{kg}$, Cayman Chemicals) 30 minutes prior to OVA-challenge. The doses of antagonists in the present study were selected according to previous reports. ${ }^{12,13}$ Animals were sacrificed 2 hours after OVA challenge by $\mathrm{CO}_{2}$ inhalation and exsanguination.

\section{Tissue preparation}

Esophageal segments were fixed in 10\% formalin and embedded in paraffin. Sections of $5 \mu \mathrm{m}$ were cut, mounted on Superfrost Plus glass slides (Fisher Scientific, Pittsburgh, PA, USA), and stained with hematoxylin-eosin (HE). For immunofluorescent staining, the esophagus was first fixed in $4 \%$ formaldehyde in PBS overnight at $4^{\circ} \mathrm{C}$. The tissues were rinsed in PBS and cryoprotected with $18 \%$ sucrose in PBS for 18-24 hours. The tissues were then covered with optimal cutting temperature mounting medium and frozen on dry ice. The segments of the esophagus were cut in serial cross-sections of $12 \mu \mathrm{m}$ thickness using a cryostat, collected on silane-coated slides, and air-dried for 30 minutes. 


\section{Immunofluorescent staining of MBP}

Immunofluorescent staining was performed according to the method described earlier. ${ }^{11,14}$ Briefly, sections were incubated with blocking solution containing $1 \%$ bovine serum albumin (BSA), $10 \%$ normal goat serum, and $0.1 \%$ Tween 20 in PBS for 60 minutes. The tissues were incubated $12-24$ hours at $4{ }^{\circ} \mathrm{C}$ with primary antibodies in PBS containing $0.1 \%$ Triton $\mathrm{X}-100$ and $1 \%$ BSA (PBS-TX-BSA). The primary antibody was monoclonal mouse anti-MBP (1:200; Chemicon (EMD Millipore), Billerica, MA, USA). Slides were then washed in PBS-TX-BSA and incubated with goat anti-mouse antibody labeled with Alexa Fluor 594 (diluted 1:200, Invitrogen, Grand Island, NY, USA) in PBS-TX-BSA for 2 hours at room temperature. The sections were rinsed with PBS, then with saline buffered with phosphate to $\mathrm{pH}$ 8.6, and coverslipped. The slides were analyzed and counted using a conventional epifluorescence microscope (Olympus BX60, Olympus Imaging America, Center Valley, PA, USA) with the filters set to allow separate visualization of Alexa 594 (rhodamine; Invitrogen) in a computer-aided digital imaging system. The stained slides were briefly checked under an epifluorescence microscope, and three well-stained cross-sections from each esophageal specimen were randomly selected for counting and averaging. Positive stainings were identified as having pixel intensity above background fluorescence.

\section{Data analysis}

In HE staining, eosinophils in the cross-sections of the esophagus were counted and averaged from three high-power fields $(400 \times)$ under light microscope. In immunostaining, the total numbers of MBP-positive eosinophils were counted and averaged from three cross-sections of each esophageal specimen. Values were expressed as mean \pm standard error of the mean. Differences between the values were determined by Student's $t$-test, and $P<0.05$ was considered statistically significant.

\section{RESULTS}

\section{Effect of PGD2 on eosinophil trafficking into the esophagus}

In naïve guinea pigs, PGD2 injection into the esophagus increased eosinophil infiltration in esophageal epithelium, as revealed by HE staining at injection site (PGD2 vs. PBS: $25 \pm 1.7 / \mathrm{mm}^{2}$ vs. $7.7 \pm 1.0 / \mathrm{mm}^{2}$, $P<0.01, n=3)$. This increased infiltration was a localized phenomenon, as no increased infiltration of eosinophils was observed in the tissues either $3 \mathrm{~mm}$ above $\left(5.3 \pm 1.3 / \mathrm{mm}^{2}\right)$ or below $\left(5.3 \pm 0.3 / \mathrm{mm}^{2}\right)$ the injection site $(P<0.05, n=3)$ (Fig. 1). The affected tissue demonstrated elongation of subepithelial papillae ( $\%$ of mucosal thickness: $58 \pm 4 \%$, vs. control at $44 \pm 13 \%, P<0.05, n=3)$. There was no obvious infiltration of other inflammatory cells (such as neutrophils) at the injection site. The infiltration of eosinophils in the esophagus was further confirmed by the staining of MBP-labeled immunoreactive (MBP-LI) cells in the esophageal epithelium (17 $\pm 2.7 /$ cross-section, vs. control of $6.3 \pm 1.3 /$ crosssection, $P<0.05, n=3$ ).

\section{Effects of DP1 and DP2 agonists on eosinophil trafficking into the esophagus}

To determine the exact roles of DP1 and DP2 on PGD2-induced eosinophil trafficking into the esophagus, either DP1 or DP2 agonist was injected into the wall of the esophagus of naïve animals in the same way as PGD2 injection. Two hours after injections, esophageal tissues were processed for immunostaining of eosinophil MBP. Injection with the DP2 agonist 15(R)-PGD2 significantly increased MBP-positive eosinophils in esophageal epithelium (DP2 agonist vs. PBS: $25 \pm 4.8 /$ cross-section vs. $6.3 \pm 1.3 /$ cross-section, $P<0.05, n=3$ ) (Fig. 2A,B). In contrast, injection with the DP1 agonist BW 245C did not significantly increase MBP-positive eosinophils in esophageal epithelium (DP1 agonist vs. PBS: $9.6 \pm 1.6 /$ crosssection vs. $6.3 \pm 1.3 /$ cross-section, $P>0.05, n=3$ ) (Fig. 2A,C). These results that DP2 agonist, but not DP1 agonist, mimicked PGD2-induced eosinophil infiltration in the esophagus suggest an important role of DP2 receptor in PGD2-induced eosinophil trafficking into the esophagus.

\section{Effect of DP1 and DP2 antagonists on OVA challenge-induced eosinophil trafficking into the esophagus}

In OVA-sensitized guinea pigs, allergen (OVA) challenge by inhalation of nebulized OVA $(0.1 \%)$ caused an allergic response resulting in gasping and increased respiratory rate within 1-5 minutes in all studied animals $(n=3)$. Animals, if pretreated with the DP2 antagonist BAY-u3405 $(n=3)$, did not develop such allergic responses during the time of OVA challenge. Similarly, no allergic response was observed in control animals (inhaled nebulized $0.9 \%$ saline in OVA-sensitized guinea pigs, $n=3$ ). However, animals pretreated with the DP1 antagonist BW 868C $(n=3)$ developed the signs of allergic responses within 5 minutes.

The total numbers of MBP-positive eosinophils were counted and compared in cross-sections of the esophagus from different treatment groups: OVAsensitized (OVA-S) animals receiving saline inhalation (as control), OVA-S animals receiving OVA challenge (OVA-C), OVA-S animals receiving DP1 


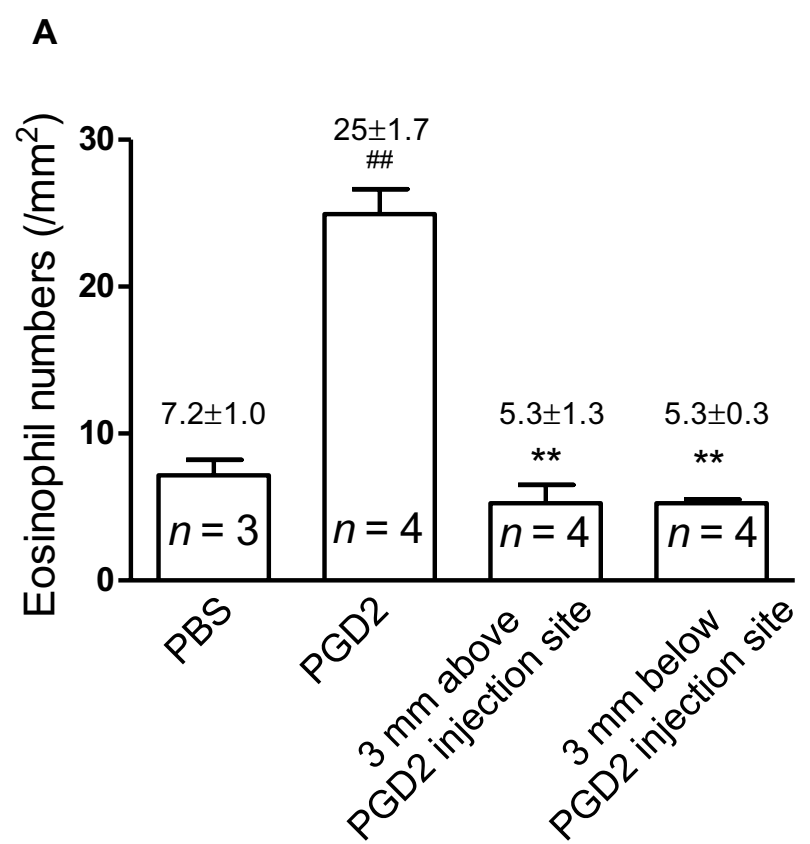

\#\# $P<0.01$ versus PBS; ${ }^{* *} P<0.01$ versus PGD2

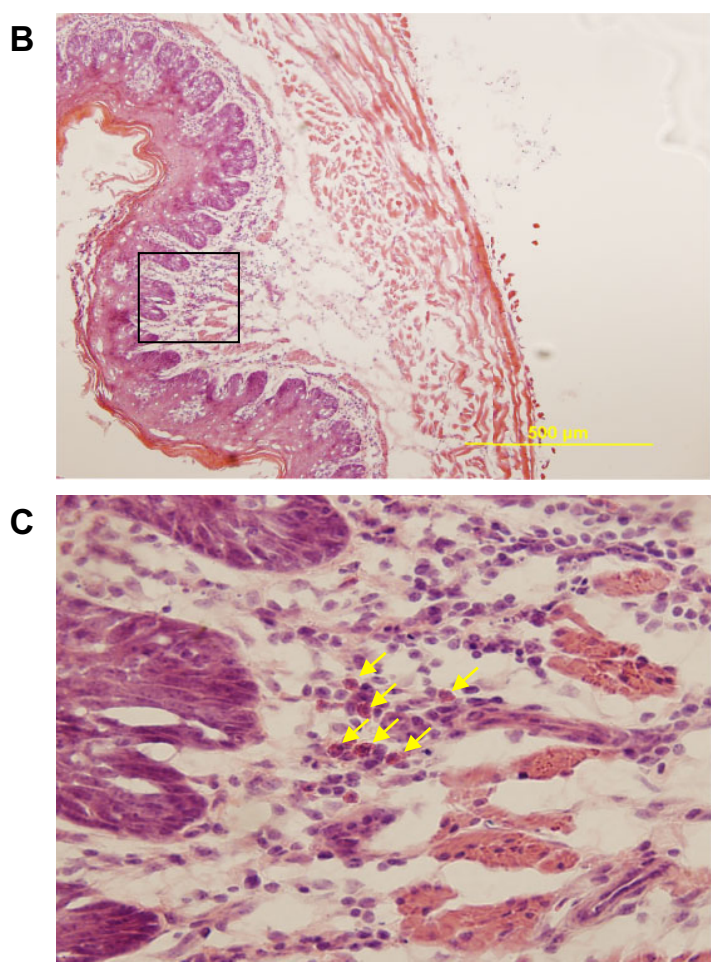

Fig. 1 (A) Prostaglandin D2 (PGD2) on eosinophil trafficking into the esophagus. PGD2 injection into the wall of the esophagus induces a localized increase of eosinophil infiltration in the esophagus. Compared with control with phosphate-buffered saline (PBS) injection, PGD2 injection significantly increases eosinophil infiltration in the esophagus as counted in hematoxylin-eosin (HE)-stained esophageal cross-sections (PBS vs. PGD2: $7.2 \pm 1.0$ vs. $25 \pm 1.7 / \mathrm{mm}^{2}, P<0.01, n=3-4$ ). Such increase is not observed either $3 \mathrm{~mm}$ above or below the injection site. (B) Esophageal cross-section under HE staining. (C) High-power field of HE staining, highlighting infiltrated eosinophils (arrows) from esophageal cross-section.

antagonist pretreatment followed by OVA-C, and OVA-S animals receiving DP2 antagonist pretreatment followed by OVA-C. In control animals (OVA-S, plus $0.9 \%$ saline inhalation), the total number of MBP-positive eosinophils in the esophagus was $6.5 \pm 0.5$ cells/cross-section $(n=3)$. The total number of MBP-positive eosinophils was significantly increased to $36.3 \pm 0.9$ cells/cross-section in the OVA-C group ( $n=3$, vs. control, $P<0.01$ ). Pretreatment with the DP2 antagonist BAY-u3405, significantly inhibited OVA challenge-induced eosinophil infiltration in the esophagus (BAY-u3405+ OVA-C vs. OVA-C: $12.6 \pm 1.9$ cells/cross-section vs. $36.3 \pm 0.9$ cells/cross-section, $P<0.01, n=3$ ) versus controls. In contrast, pretreatment with DP1 antagonist BW A868C did not significantly inhibit eosinophil infiltration in the esophagus induced by OVA challenge (BW 868C + OVA-C vs. OVA-C: $29.0 \pm$ 3.7 cells/cross-section vs. $36.3 \pm 0.9$ cells/cross-section, $P>0.05, n=3$ ) (Fig. 3). These data support our hypothesis that DP2 mediates PGD2-induced eosinophil trafficking into the esophagus.

\section{DISCUSSION}

PGD2 is an important inflammatory mediator that not only participates in mast cell activation-induced type I hypersensitivity including smooth muscle contraction, vascular leak, and vasodilation, but also displays potent chemotactic effects on eosinophils, basophils, and Th2 cells. In addition, it may potentiate inflammatory responses induced by other relevant mediators. Although PGD2 has been shown to be present in the esophagus, its physiological function and role in esophageal disorders are still largely unknown.

PGD2 is mainly synthesized and released from activated mast cells. The biological effects of PGD2 are usually mediated by its two G-protein-coupled receptors: DP1 and DP2. The DP1 receptor is more widely expressed in leukocytes, vasculature, the central nervous system, retina, lung, and intestine. The DP2 receptor is predominately expressed in eosinophils, basophils, and Th2 cells, and mediates the PGD2-induced chemotactic effect. ${ }^{15,16}$ The chemotactic effect of PGD2 on eosinophils was first reported in mice deficient in PGD2 receptor (DP receptor). Sensitization and aerosol challenge of DP-deficient mice with OVA leads to great reduction of Th2 cytokines and marginal eosinophil infiltration in the lung, with animals failing to develop airway hyperreactivity. ${ }^{17}$ This was followed by the discovery of a novel PGD2 receptor, CRTH2, which also shows to play an important role in mediating PGD2induced chemotactic effects on eosinophils. ${ }^{18,19}$ These 
A

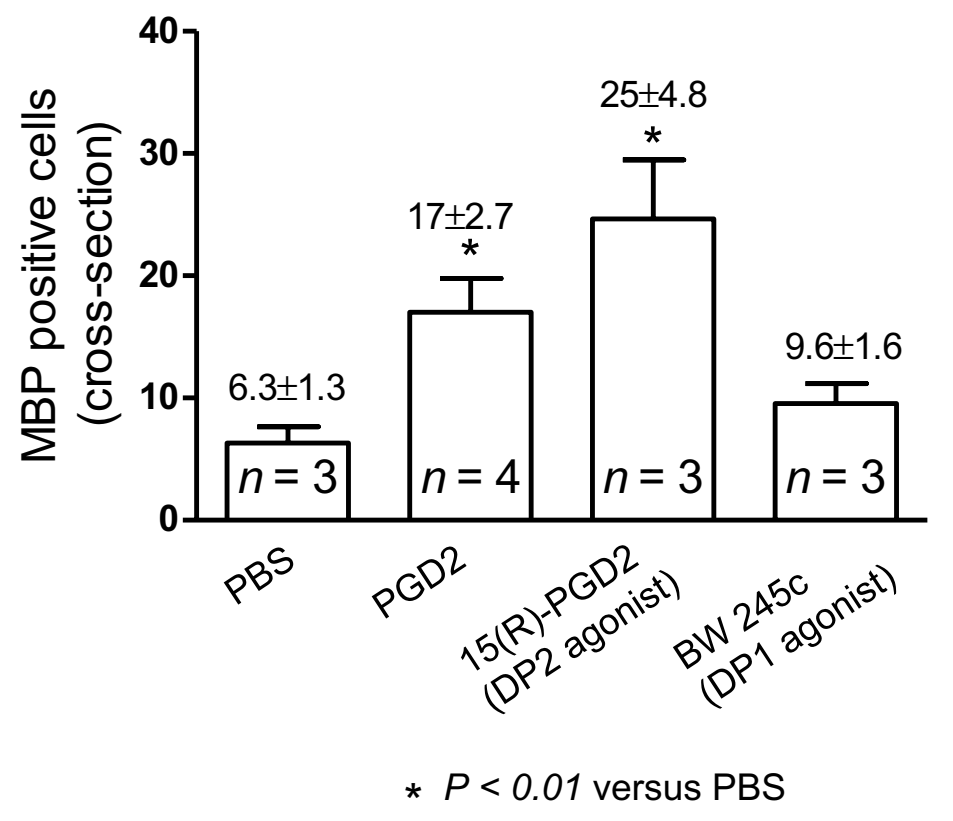

B

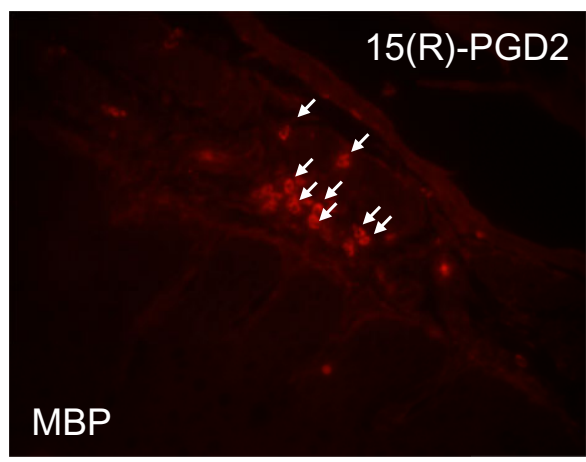

C

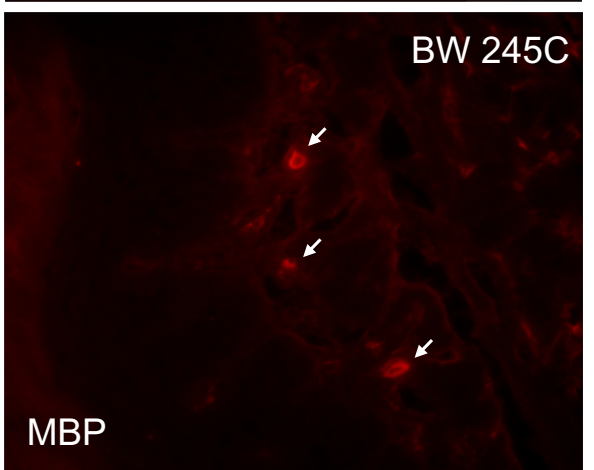

Fig. 2 Prostaglandin D2 (PGD2), DP1 agonist, and DP2 agonists on eosinophil trafficking into the esophagus. (A) Injection with PGD2 increases major basic protein (MBP)-positive-labeled eosinophils in the esophagus. This effect is mimicked by DP2 agonist 15(R)-PGD2, but not by DP1 agonist BW 245C. (B) Immunofluorescent staining of eosinophil MBP in esophageal cross-section after esophageal injection of DP2 agonist 15(R)-PGD2; (C) Immunofluorescent staining of MBP in esophageal cross-section after esophageal injection of DP1 agonist BW 245C.

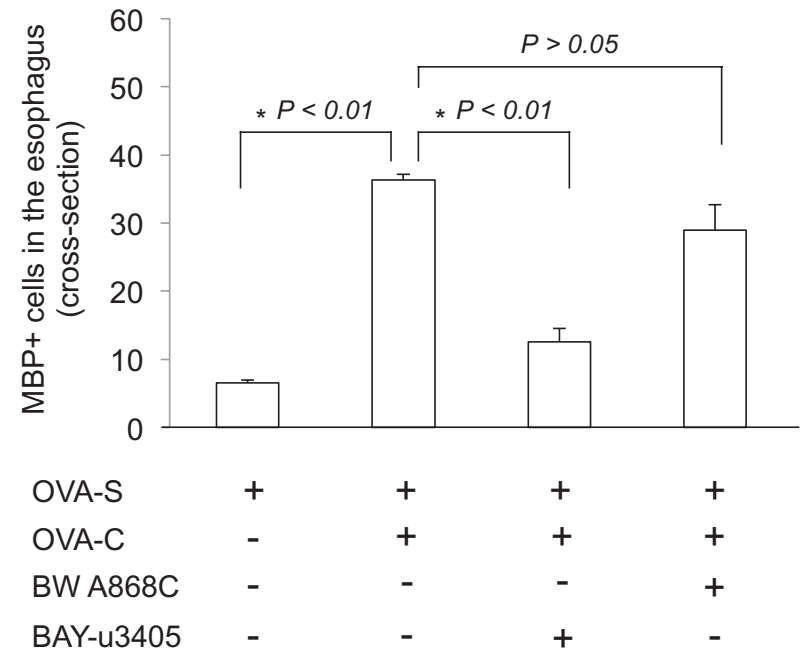

Fig. 3 Prostaglandin D2 (PGD2) DP1 and DP2 antagonists on eosinophil infiltration in the esophagus. In ovalbumin (OVA)sensitized guinea pigs, OVA challenge significantly increases MBP-positive-labeled eosinophils in the esophagus (OVA-S vs. OVA-S + OVA-C: $6.5 \pm 0.5$ vs. $36.3 \pm 0.9 /$ cross-section, $P<0.01, n=3)$. This effect can be prevented by pretreatment with DP2 antagonist BAY-u3405 (5 mg/kg, iv) (OVA-S + OVA-C vs. BAY-u3405 + OVA-S + OVA-C: $36.3 \pm 0.9$ vs. $12.6 \pm 1.6 /$ cross-section, $P<0.01, n=3$ ), but not by DP1 antagonist BW A868C (100 $\mu \mathrm{g} / \mathrm{kg}$, iv) (OVA-S + OVA-C vs. BW A868C + OVA-S + OVA-C: $36.3 \pm 0.9$ vs. $29.0 \pm 3.7 /$ cross-section, $P>0.05, n=3$ ). two PGD2 receptors DP and CRTH2 are now classified as DP1 and DP2. ${ }^{20}$

Recent studies revealed that PGD2 receptors play important roles in mediating eosinophil migration and infiltration in the peripheral tissues, such as the airway $^{13,21}$ and skin. ${ }^{22,23}$ Accumulated evidence consistently supports a predominant role of DP2 in PGD2induced chemotactic effect on eosinophils. ${ }^{24-28}$ But it is still unclear whether mast cell PGD2 also induces eosinophil trafficking into the esophagus. The present study provides the first evidence that PGD2 induces eosinophil trafficking into the esophagus, that this chemotactic effect is mimicked by DP2 agonist and that it can be prevented by DP2 antagonist, suggesting an important role for DP2 in this process. This is in agreement with the aforementioned observations in the skin and airway that under allergic and inflammatory conditions, PGD2 released from mast cells plays a predominant role in recruiting eosinophils into the peripheral tissues.

OVA inhalation in OVA-sensitized animals leads to type I hypersensitivity and specifically induces mast cell activation. This model has been widely applied as an asthma model to study allergic response in the airway. EoE is usually accompanied with an allergic condition such as food allergy or aeroallergen sensitization. ${ }^{29}$ We believe that the data from the asthma model are of relevance to EoE, especially when investigating the specific roles of mast cells and 
PGD2 on eosinophil trafficking in the tissue. In the present study, antagonist effect was determined by using in vivo guinea pig model as we previously described. ${ }^{11}$ This model has inherent limitations and cannot rule out whether other mediators and factors, such as histamines, Th2 cytokines, and eotaxin, may also have roles in eosinophil trafficking into the esophagus. Based on the present observations, two important considerations need to be clarified. First, whether the expression of eotaxin increases in the esophagus after local injections with PGD2 and DP2 agonist. Second, whether the DP2 antagonist BAYu3405 dose dependently inhibits eosinophil infiltration in the esophagus induced by OVA inhalation. Additionally, further studies using PGD2 receptor knockout mice with more localized antigen challenge in the esophagus could help to better understand the underlying mechanism of PGD2-induced eosinophil trafficking into the esophagus.

In summary, the present study for the first time demonstrated that PGD2 induces eosinophil trafficking into the esophagus. This chemotactic effect is mediated by DP2 receptor, suggesting a potential role of DP2 antagonist in the prevention of EoE.

\section{Acknowledgment}

We thank Professor Jim Potter (Johns Hopkins University School of Medicine) for the edit and comment on the manuscript.

\section{References}

1 Liacouras C A, Furuta G T, Hirano I et al. Eosinophilic esophagitis: updated consensus recommendations for children and adults. J Allergy Clin Immunol 2011; 128: 3-20.

2 Mueller S, Aigner T, Neureiter D, Stolte M. Eosinophil infiltration and degranulation in oesophageal mucosa from adult patients with eosinophilic oesophagitis: a retrospective and comparative study on pathological biopsy. J Clin Pathol 2006; 59: 1175-80

3 Kato M, Kephart G M, Talley N J et al. Eosinophil infiltration and degranulation in normal human tissue. Anat Rec 1998; 252: 418-25.

4 Allende D S, Yerian L M. Diagnosing gastroesophageal reflux disease: the pathologist's perspective. Adv Anat Pathol 2009; 16: 161-5. Review.

5 Straumann A, Conus S, Grzonka P et al. Anti-interleukin-5 antibody treatment (mepolizumab) in active eosinophilic oesophagitis: a randomised, placebo-controlled, double-blind trial. Gut 2010; 59: 21-30.

6 Gonsalves N, Yang G Y, Doerfler B, Ritz S, Ditto A M, Hirano I. Elimination diet effectively treats eosinophilic esophagitis in adults; food reintroduction identifies causative factors. Gastroenterology 2012; 142: 1451-9.

7 Kirsch R, Bokhary R, Marcon M A, Cutz E. Activated mucosal mast cells differentiate eosinophilic (allergic) esophagitis from gastroesophageal reflux disease. J Pediatr Gastroenterol Nutr 2007; 44: 20-6.

8 Konikoff M R, Noel R J, Blanchard C et al. A randomized, double-blind, placebo-controlled trial of fluticasone propionate for pediatric eosinophilic esophagitis. Gastroenterology 2006; 131: 1381-91.

9 Straumann A, Bauer M, Fischer B, Blaser K, Simon H U. Idiopathic eosinophilic esophagitis is associated with a
$\mathrm{T}(\mathrm{H}) 2$-type allergic inflammatory response. J Allergy Clin Immunol 2001; 108: 954-61.

10 Abonia J P, Blanchard C, Butz B B et al. Involvement of mast cells in eosinophilic esophagitis. J Allergy Clin Immunol 2010; 126: 140-9.

11 Yu S, Stahl E, Li Q, Ouyang A. Antigen inhalation induces mast cells and eosinophils infiltration in the guinea pig esophageal epithelium involving histamine-mediated pathway. Life Sci 2008; 82: 324-30.

12 Hamid-Bloomfield S, Whittle B J. Antagonism of PGD2 vasodepressor responses in the rat in vivo by the novel, selective antagonist, BW A868C. Br J Pharmacol 1989; 96: 307-12.

13 Shiraishi Y, Asano K, Nakajima T et al. Prostaglandin D2-induced eosinophilic airway inflammation is mediated by CRTH2 receptor. J Pharmacol Exp Ther 2005; 312: 954-60.

14 Yu S, Kollarik M, Ouyang A, Myers A C, Undem B J. Mast cell-mediated long-lasting increases in excitability of vagal $\mathrm{C}$ fibers in guinea pig esophagus. Am J Physiol Gastrointest Liver Physiol 2007; 293: G850-6.

15 Pettipher R. The roles of the prostaglandin $\mathrm{D}(2)$ receptors $\mathrm{DP}(1)$ and $\mathrm{CRTH} 2$ in promoting allergic responses. $\mathrm{Br} \mathrm{J}$ Pharmacol 2008; 153 (Suppl 1): S191-9. Review.

16 Schuligoi R, Sturm E, Luschnig P et al. CRTH2 and D-type prostanoid receptor antagonists as novel therapeutic agents for inflammatory diseases. Pharmacology 2010; 85: 372-82. Review.

17 Matsuoka T, Hirata M, Tanaka H et al. Prostaglandin D2 as a mediator of allergic asthma. Science 2000; 287: 2013-17.

18 Hirai H, Tanaka K, Yoshie O et al. Prostaglandin D2 selectively induces chemotaxis in T helper type 2 cells, eosinophils, and basophils via seven-transmembrane receptor CRTH2. J Exp Med 2001; 193: 255-61.

19 Monneret G, Gravel S, Diamond M, Rokach J, Powell W S. Prostaglandin D2 is a potent chemoattractant for human eosinophils that acts via a novel DP receptor. Blood 2001; 98: 1942-8.

20 Woodward D F, Jones R L, Narumiya S. International Union of Basic and Clinical Pharmacology. LXXXIII: classification of prostanoid receptors, updating 15 years of progress. Pharmacol Rev 2011; 63: 471-538. Review.

21 Almishri W, Cossette C, Rokach J, Martin J G, Hamid Q, Powell W S. Effects of prostaglandin D2, 15-deoxy-Delta12,14prostaglandin J2, and selective DP1 and DP2 receptor agonists on pulmonary infiltration of eosinophils in Brown Norway rats. J Pharmacol Exp Ther 2005; 313: 64-9.

22 Satoh T, Moroi R, Aritake K et al. Prostaglandin D2 plays an essential role in chronic allergic inflammation of the skin via CRTH2 receptor. J Immunol 2006; 177: 2621-9.

23 Spik I, Brénuchon C, Angéli V et al. Activation of the prostaglandin D2 receptor DP2/CRTH2 increases allergic inflammation in mouse. J Immunol 2005; 174: 3703-8.

24 He R, Oyoshi M K, Wang J Y, Hodge M R, Jin H, Geha R S. The prostaglandin D2 receptor CRTH2 is important for allergic skin inflammation after epicutaneous antigen challenge. J Allergy Clin Immunol 2010; 126: 784-90.

25 Schratl P, Royer J F, Kostenis E et al. The role of the prostaglandin D2 receptor, DP, in eosinophil trafficking. J Immunol 2007; 179: 4792-9.

26 Shichijo M, Sugimoto H, Nagao K et al. Chemoattractant receptor-homologous molecule expressed on Th2 cells activation in vivo increases blood leukocyte counts and its blockade abrogates 13,14-dihydro-15-keto-prostaglandin D2-induced eosinophilia in rats. J Pharmacol Exp Ther 2003; 307: 51825 .

27 Sugimoto $\mathrm{H}$, Shichijo M, Iino T et al. An orally bioavailable small molecule antagonist of $\mathrm{CRTH} 2$, ramatroban (BAY u3405), inhibits prostaglandin D2-induced eosinophil migration in vitro. J Pharmacol Exp Ther 2003; 305: 347-52.

28 Yamamoto Y, Otani S, Hirai H et al. Dual functions of prostaglandin D2 in murine contact hypersensitivity via DP and CRTH2. Am J Pathol 2011; 179: 302-14.

29 Straumann A, Aceves S S, Blanchard C et al. Pediatric and adult eosinophilic esophagitis: similarities and differences. Allergy 2012; 67: 477-90. 